\title{
A modified pectoralis major myocutaneous flap technique with improved vascular supply and an extended rotation arc for oral defects: A case report
}

\author{
TAKAHIRO KANNO, YOSHIKI NARIAI, HIROTO TATSUMI, MASAAKI KARINO, \\ AYA YOSHINO and JOJI SEKINE
}

Department of Oral and Maxillofacial Surgery, Shimane University Faculty of Medicine, Izumo, Shimane 693-8501, Japan

Received November 20, 2014; Accepted July 7, 2015

DOI: $10.3892 / 01.2015 .3696$

\begin{abstract}
The pedicled pectoralis major myocutaneous (PMMC) flap is versatile, and is widely used for the treatment of surgical defects following oral cancer resection. Although free-tissue transfer of a vascularized free flap is often preferred, the clinical benefits of the PMMC flap should not be overlooked. The conventional technique of harvesting a PMMC flap involves a single vascular supply from the pectoral branch of the thoracoacromial artery. However, this approach compromises the distal skin island of the flap, and requires an indirect blood supply via communicating vessels, which increases the potential risk of partial distal flap necrosis. When harvesting a PMMC flap for oral and maxillofacial reconstruction, preservation of the lateral thoracic artery and use of the subclavian route are alternatives that ensure sufficient blood supply and an increased rotation arc. Such an approach enables the harvesting of a PMMC flap that can reach the entire oral cavity, including the infraorbital region, palate, middle pterygopalatine fossa and nasopharynx, with no risk of vascular insufficiency to the distal skin island. In conclusion, the technique described in the present study was able to improve the blood supply of the distal PMMC flap and increase its rotation arc.
\end{abstract}

\section{Introduction}

Due to its versatility, the pedicled pectoralis major myocutaneous (PMMC) flap is extensively used for the treatment of surgical defects resulting from oral and maxillofacial cancer ablation (1). Despite the fact that microvascularized free-tissue transfer is increasingly becoming the main flap method for

Correspondence to: Dr Takahiro Kanno, Department of Oral and Maxillofacial Surgery, Shimane University Faculty of Medicine, 89-1 Enyacho, Izumo, Shimane 693-8501, Japan

E-mail: tkanno@med.shimane-u.ac.jp

Key words: pectoralis major myocutaneous flap, oral cancer, reconstruction, blood supply, rotation arc oral, head and neck cancer reconstruction, this method cannot be used effectively in certain situations, including recurrence following reconstruction (salvage), in the presence of poor systemic or local conditions, following full-dose radiotherapy, in patients with arteriosclerosis or in the elderly $(1,2)$.

Numerous clinicians have criticized the PMMC flap due to its relatively high rate of fistula formation, bulkiness of the muscle pedicle and adipose layer, disfiguring donor-site morbidity, and relatively high potential (15-32\%) for distal or marginal flap necrosis with partial flap loss, as a result of vascular insufficiency $(1,3)$. In addition, the limited arc of rotation results in reduced flexibility following oral and maxillofacial, or head and neck reconstruction, compared with free-tissue transfer methods $(2,3)$. The PMMC flap was initially described by Ariyan (4) in 1979, and has since been used for salvage and advanced primary reconstruction, with numerous technical modifications based on 3-dimensional blood circulation $(2,5)$.

Kiyokawa et al (5) and Rikimaru et al (6) improved the restricted range of the PMMC flap rotation arc by developing a novel method of flap elevation and transfer, in order to extend its range and degree of flexibility whilst maintaining stable blood circulation in the skin paddle. This was achieved by moving the flap to the reconstruction site via the subclavian route, which provides a high degree of flexibility with a greater rotation arc and $\sim 8 \mathrm{~cm}$ extension, compared with the original method described by Ariyan (4).

A further drawback of the PMMC flap is vascular insufficiency due to unstable blood supply, which may result in the partial loss of the distal and marginal parts of the flap. This is particularly a problem when the flap is harvested from the caudal vascular territory (far caudal from the fourth costal cartilage to the seventh) through the choke vessels of communicating vessels in an axial pattern based on the angiosome concept $(2,3,6)$. As a result, unreasonable tension in the skin paddle may lead to partial distal or marginal necrosis (5). Kiyokawa et al (5) and Rikimaru et al (6) partially resolved this problem by designing a skin paddle that included a large number of perforating branches, which is suitable regardless of the size of the flap. Studies of 3-dimensional blood circulation and cadaver anatomy have demonstrated that preservation of the lateral thoracic artery, in addition to the conventional 
technique for head and neck reconstruction, stabilizes the blood supply to the distal (caudal) part of the flap without compromising pedicle length $(2,6)$.

In the present study, a simple modification of the conventional PMMC flap harvesting method, which is able to overcome the weaknesses of the flap, is reported. In the modified method, the blood supply is secured by preserving the lateral thoracic vessels and using the subclavian route. In addition, the rotation arc is increased for oral and maxillofacial reconstruction.

\section{Case report}

Study approval. The present study was approved by the Ethical Committee of Shimane University Faculty of Medicine (Izumo, Shimane, Japan) (study no. 1522). Written informed consent for publication of the clinical images was provided by the patient.

Patient presentation. The patient, a 57-year-old Japanese female affected by oral cancer, was admitted to the Department of Oral and Maxillofacial Surgery of Shimane University Hospital (Izumo, Japan) on December $19^{\text {th }}$ 2013, and subjected to PMMC flap reconstruction.

Surgical technique. When harvesting the PMMC flap, the pectoralis major muscle was elevated from the chest wall and moved upward, as in the conventional surgical technique (Fig. 1A). The lateral thoracic vessels were identified beneath the lateral border of the pectoralis major muscle in the region of the axilla. The pectoral part of the pectoralis major muscle was divided lateral to the lateral thoracic artery, and medial to the pectoral branch of the thoracoacromial artery up to the clavicular part of the muscle. The clavicular part of the pectoralis major muscle overlying the pectoral branch of the thoracoacromial artery was then divided up to the clavicle using the true-island PMMC flap technique described by Wei et al (7). In the conventional flap preparation technique, the lateral thoracic artery, which runs upward beneath the lateral border of the pectoralis minor muscle, is sacrificed at this stage, since it limits the length of the flap pedicle. The pectoralis minor muscle may be divided around the lateral thoracic vessels to release them, as depicted in Fig. 1B. Subsequently, the clavicular periosteum around the pectoral branches of the thoracoacromial vessels was excised from the cervical and thoracic sides, and the periosteum on the inferior surface of the clavicle was detached and reflected in order to drop downward, as described by Kiyokawa et al (5). This procedure enables the safe transfer of the PMMC flap and its rotation toward the oral and maxillofacial region under the clavicle without damaging the subclavian vessels (Fig. 1C). Furthermore, it enables the PMMC flap to safely reach the entire oral cavity, including the infraorbital region, palate, middle pterygopalatine fossa and nasopharynx, with no distal vascular insufficiency.

This modified surgical approach has been performed on 7 patients at the Department of Oral and Maxillofacial Surgery of Shimane University Hospital, and no post-surgery complications have been observed thus far. Regarding the surgical procedure, the double-pedicled PMMC flap reconstruction with transfer via a subclavian route was routinely followed by
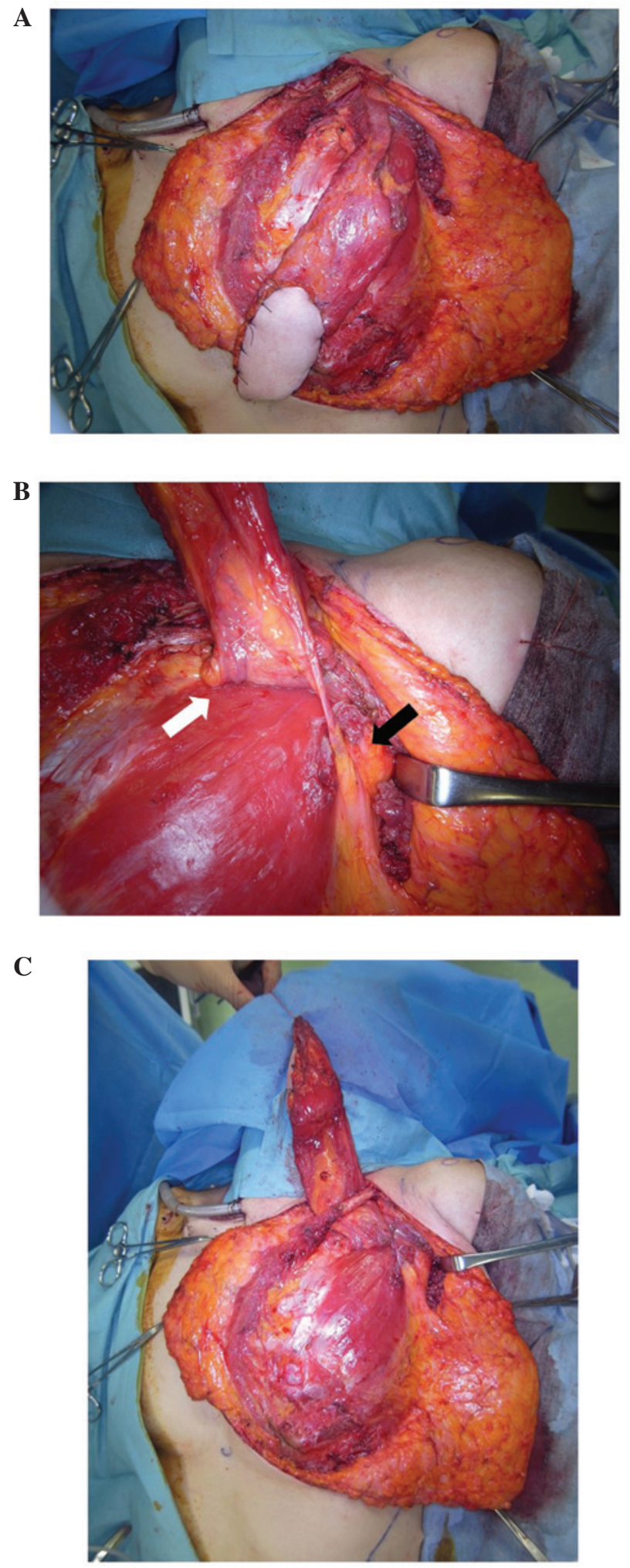

Figure 1. (A) Harvesting a pedicled pectoralis major myocutaneous (PMMC) flap with a 9x7-cm skin paddle, including the lateral thoracic vessels. (B) The pectoralis minor muscle was divided around the lateral thoracic vessels to enable the supply of blood to the PMMC flap. The white and black arrows in the image indicate the thoracoacromial and lateral thoracic vessels, respectively. (C) The entire PMMC flap was moved safely and rotated toward the recipient site under the clavicle.

tracheostomy, radical neck dissectionformetastaticlymphnodes and en bloc complete margin-free cancer ablation surgery, as in the present case (Fig. 2A and B). The postoperative adjuvant 
A

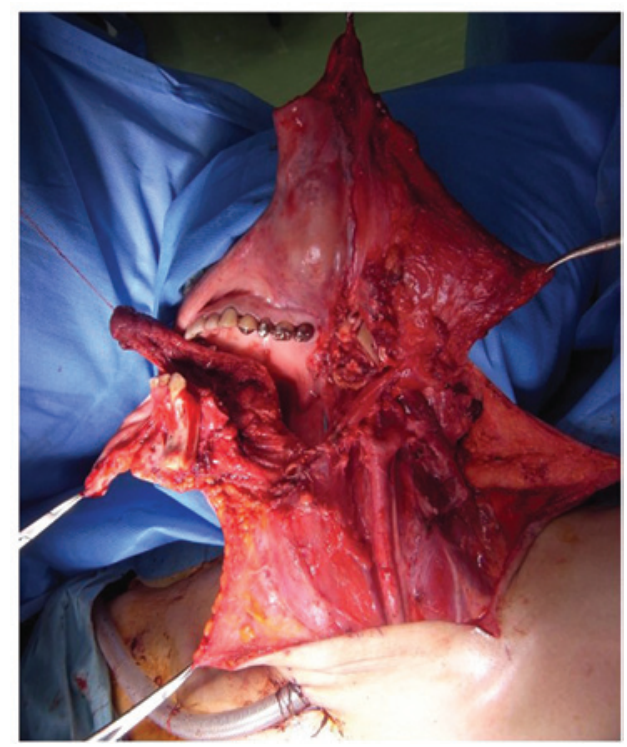

B

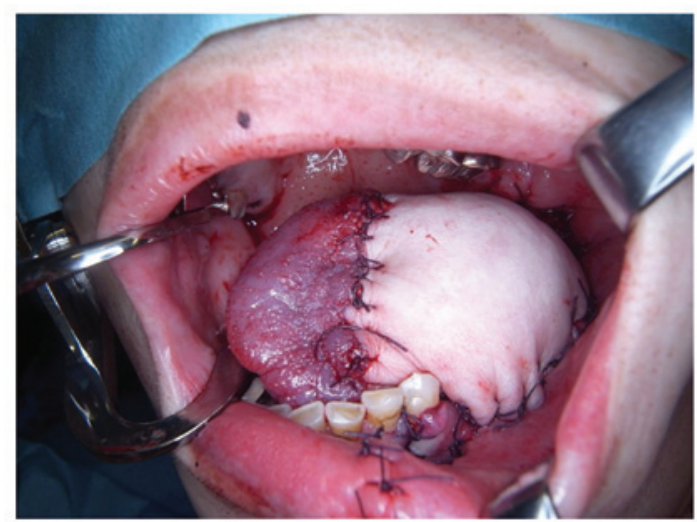

C

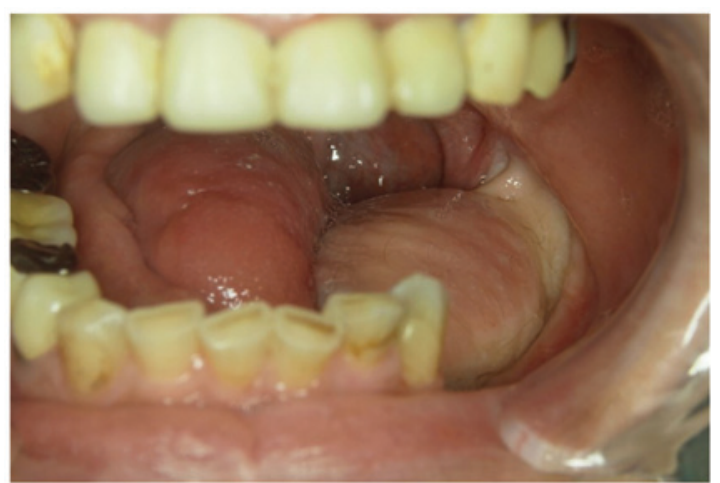

Figure 2. (A) Patient with two advanced types of cancer (tongue squamous cell carcinoma with multiple ipsilateral cervical LNMs, cT4aN2bM0 stage IVa; and rectum adenocarcinoma with LNM, cT3N2M0, stage IIIb) was subjected to pedicled PMMC flap reconstruction with a temporary mandibular reconstruction plate, followed by tracheostomy, radical neck dissection for multiple LNMs (levels I-V), en bloc complete margin-free cancer ablation surgery (comprising a subtotal glossectomy and segmental resection of the mandible) and surgical treatment of the rectum adenocarcinoma. (B) Elevated PMMC flap was transferred to the reconstruction site via a subclavian route, and water-tight closure was performed. (C) Intraoral image at 6 months post-surgery, indicating good functional tongue mobility with no fistulas. LNM, lymph node metastasis; PMMC, pectoralis major myocutaneous.

treatments were performed according to the current National Comprehensive Cancer Network Guidelines (8). The patients were subjected to regular postoperative clinical follow-up with functional assessment. In the present case, good functional tongue mobility with no fistulas was observed 6 months after surgery (Fig. 2C). However, the patient succumbed to distant metastases 9 months after undergoing surgery.

\section{Discussion}

Despite the wide clinical application of the PMMC flap in oral, head and neck reconstructive surgery, numerous PMMC flaps have been observed to develop partial distal necrosis and display limited flexibility $(1,3)$. Although the restricted range of rotation reported in the original technique was resolved with the development of the subclavian route, the blood supply to the distal (caudal) portion of the PMMC flap remained problematic $(1,5)$. Anatomical studies have aided to improve PMMC flap preparation (6). In particular, Yang et al (9) and Elazab and Nabil (10) demonstrated that the pectoral branches of the thoracoacromial trunk vessels arising from the first and second parts of the axillary artery provide the main blood supply to the skin paddle island and the overlying upper part of the pectoralis major muscle. Although the anterior intercostal branches of the internal mammary artery supply blood to $\sim 43 \%$ of the skin territory overlying the medial and lower parts of the PMMC flap, the lateral thoracic artery is often the main blood supply to the lateral and lower parts of the pectoralis major muscle, overlying the paddle skin in $\sim 6.6 \%$ of PMMC flaps $(3,9,10)$.

Previous studies have demonstrated that the distal segment of the pectoralis major muscle is supplied by the pectoral branch of the thoracoacromial, axillary and lateral thoracic artery in 90,67 and 40\% of cases, respectively $(3,9,10)$. Po-Wing Yuen (3) reported a simple technique for preserving the lateral thoracic artery during PMMC flap harvesting. In this technique, the lateral thoracic artery is identified and elevated subsequent to dividing the overlying pectoralis minor muscle, and the lateral thoracic artery and pectoralis branch of the thoracoacromial artery that is part of the double pedicled flap are then rotated upward through the supraclavian root via a subcutaneous tunnel (3). Makiguchi et al (11) developed the 'supercharged PMMC flap' technique, which preserves the lateral thoracic artery by tracing the lateral thoracic vessels upward near the bifurcation of the subclavian vessels. At this location, the vessels are sectioned and anastomosed to the cervical vessels to create a conventionally prepared PMMC flap nourished by the dominant thoracoacromial vessels. In addition, the vessels are also rotated upward through the supraclavian root via a subcutaneous tunnel, similar to that described in the modified method of Po-Wing Yuen (3). Therefore, preserving the lateral thoracic artery during PMMC flap harvesting to improve the distal blood supply is reasonable. The Department of Oral and Maxillofacial Surgery of Shimane University Hospital has been using this modified technique over the conventional microvascularized free flap reconstruction technique since 2013. To date, seven patients with advanced or recurrent oral cancer, a number of whom were elderly, have been treated with this modified technique, and no flap problems or necrosis have been observed. Since the technique described in the present study involves dividing the branches of the internal mammary and axillary arteries, 
the potential risks of partial distal flap necrosis have not been completely overcome. However, preservation of the lateral thoracic vessels, which may be major contributors of blood supply to the distal and lateral parts of the PMMC flap and skin paddle, is advantageous.

In conclusion, the modification of the PMMC flap technique described in the present study has been demonstrated to preserve the lateral thoracic vessels without limiting the rotation arc of the flap, due to the use of the subclavian route. Furthermore, this modified technique is simpler and safer than those that divide the pectoralis minor muscle, or section and anastomose the lateral thoracic vessels.

\section{Acknowledgements}

The authors of the present study would like to thank all of the participants. The present study was in part supported by Grant-in-Aid for Scientific Research (KAKENHI) and Young Scientists (B; grant no. 25870452 to Dr Takahiro Kanno).

\section{References}

1. Jena A, Patnayak R, Sharan R, Reddy SK, Manilal B and Rao LM: Outcomes of pectoralis major myocutaneous flap in female patients for oral cavity defect reconstruction. J Oral Maxillofac Surg 72: 222-231, 2014.
2. Rikimaru H, Kiyokawa K, Watanabe K, Koga N, Nishi Y and Sakamoto A: New method of preparing a pectoralis major myocutaneous flap with a skin paddle that includes the third intercostal perforating branch of the internal thoracic artery. Plast Reconstr Surg 123: 1220-1228, 2009.

3. Po-Wing Yuen A: Preservation of lateral thoracic artery to improve vascular supply of distal skin without compromising pedicle length in harvesting pectoralis major myocutaneous flap. J Plast Reconstr Aesthet Surg 59: 1433-1435, 2006.

4. Ariyan S: The pectoralis major myocutaneous flap. A versatile flap for reconstruction in the head and neck. Plast Reconstr Surg 63: 73-81, 1979.

5. Kiyokawa K, Tai Y, Tanabe HY, Inoue Y, Yamauchi T, Rikimaru H, Mori K and Nakashima T: A method that preserves circulation during preparation of the pectoralis major myocutaneous flap in head and neck reconstruction. Plast Reconstr Surg 102: 2336-2345, 1998.

6. Rikimaru H, Kiyokawa K, Inoue Y and Tai Y: Three-dimensional anatomical vascular distribution in the pectoralis major myocutaneous flap. Plast Reconstr Surg 115: 1342-1354, 2005.

7. Wei WI, Lam KH and Wong J: The true pectoralis major myocutaneous island flap: An anatomical study. Br J Plast Surg 37: $568-573,1984$.

8. National Comprehensive Cancer Network (NCCN) Clinical Practice Guidelines in Oncology: Head and Neck Cancers. Version 2. NCCN, Fort Washington, USA, pp16-20, 2013.

9. Yang D, Marshall G and Morris SF: Variability in the vascularity of the pectoralis major muscle. J Otolaryngol 32: 12-15, 2003.

10. Elazab EEB and Nabil NM: Pectoralis major muscle: Anatomical features of its arterial supply. Eur J Plast Surg 35: 9-18, 2012.

11. Makiguchi T, Yokoo S, Miyazaki H, Takayama Y, Ogawa M, Hashikawa K and Terashi H: Supercharged pectoralis major musculocutaneous flap. J Craniofac Surg 24: e179-e182, 2013. 